Article

\title{
Rational Savings Account Models for Backward-Looking Interest Rate Benchmarks
}

\author{
Andrea Macrina ${ }^{1,2, *}$ and David Skovmand ${ }^{3}$ (D) \\ 1 Department of Mathematics, University College London, London WC1E 6BT, UK \\ 2 African Institute for Financial Markets and Risk Management, University of Cape Town, \\ Rondebosch 7701, South Africa \\ 3 Department of Mathematics, University of Copenhagen, 2100 Copenhagen, Denmark; \\ skovmand@math.ku.dk \\ * Correspondence: a.macrina@ucl.ac.uk
}

Received: 20 January 2020; Accepted: 24 February 2020; Published: 3 March 2020

check for updates

\begin{abstract}
Interest rate benchmarks are currently undergoing a major transition. The LIBOR benchmark is planned to be discontinued by the end of 2021 and superseded by what ISDA calls an adjusted risk-free rate (RFR). ISDA has recently announced that the LIBOR replacement will most likely be constructed from a compounded running average of RFR overnight rates over a period matching the LIBOR tenor. This new backward-looking benchmark is markedly different when compared with LIBOR. It is measurable only at the end of the term in contrast to the forward-looking LIBOR, which is measurable at the start of the term. The RFR provides a simplification because the cash flows and the discount factors may be derived from the same discounting curve, thus avoiding —on a superficial level—any multi-curve complications. We develop a new class of savings account models and derive a novel interest rate system specifically designed to facilitate a high degree of tractability for the pricing of RFR-based fixed-income instruments. The rational form of the savings account models under the risk-neutral measure enables the pricing in closed form of caplets, swaptions and futures written on the backward-looking interest rate benchmark.
\end{abstract}

Keywords: LIBOR; SOFR; SONIA; LIBOR transition; risk-free rates; rational term structure models; swaptions; caplets; futures

\section{Introduction}

Since the speech of Andrew Bailey, the Chief Executive of the Financial Conduct Authority, in 2017 on the future of the London interbank offered rate (LIBOR) it has steadily become clearer that the LIBOR interest rate benchmarks are no longer going to be supported in several major currency denominations. The change is primarily justified by the observation that unsecured (term-) lending, the cost of which LIBOR is meant to measure, is no longer a significant source of financing for financial institutions. Furthermore, the current LIBOR benchmark has fallen out favour, i.a., due to its survey-based nature prone to manipulation, see e.g., Gyntelberg and Wooldridge (2008).

The move, much advocated by regulators suchlike the Financial Conduct Authority (FCA), has been towards a benchmark detached from the subjective opinions of a panel, such as the LIBOR panel of global banks, and closer to actual financial transactions. It has been decided, see ISDA (2019), that reliance on LIBOR and most of the other IBOR benchmarks should end and a transaction-based overnight rate - a so-called risk-free rate (RFR) - introduced as the alternative. In the USA, the Secured Overnight Financing Rate (SOFR) — an overnight rate derived from repurchase agreement transactions-is the chosen RFR. A similar benchmark, the reformed Sterling Overnight Index Average (SONIA), has been chosen in the UK, while in the Eurozone the Euro Short-Term Rate (€STR) is 
the preferred RFR, which is to supersede EURIBOR ${ }^{1}$. By construction, IBORs and the RFRs do not capture/reflect the same risks, see Backwell et al. (2019) for a discussion of this central discrepancy between overnight and term-based benchmarks. Even if they did, LIBOR is a term rate and therefore cannot be replaced as such by an overnight rate. A transformation of the RFR is thus necessary in order to achieve a certain level of affinity with the current LIBOR benchmark. After consulting a panel of market participants, the International Swaps and Derivatives Association (ISDA) has recently reported, c.f. ISDA (2019), that the preferred LIBOR replacement will be based on a discretely compounded average of daily rates, averaged over a period matching the LIBOR tenors, it is to supplant. Such a successor rate is known as a backward-looking benchmark. Furthermore, to account for differences in the risk premia associated with LIBOR and the RFR, respectively, a constant spread will be added to the backward-looking benchmark that will be based on historical data. The details of its determination can be found in ISDA (2019).

LIBOR is expected to be fully discontinued by the end of 2021. However, it is still (at the time of writing) the main benchmark newly issued loans and derivatives are linked to. In May 2018, the Chicago Mercantile Exchange (CME) launched SOFR and SONIA futures on their trading platform, and, while trading in these contracts is still sparse, the volume is growing. One would expect other linear and non-linear SOFR derivatives to follow suit as the 2021 deadline nears. Taking the current LIBOR-based derivatives market as an indicator, one would expect RFR linked versions of swaps, caps and swaptions along with the already trading SOFR/SONIA futures to become liquid once LIBOR has been discontinued.

In this paper we develop a class of discounting models that allows for closed-form pricing of derivatives written on a backward-looking benchmark. It is motivated by the recent work of Mercurio (2018) and Lyashenko and Mercurio (2019). The former presents a short-rate model for SOFR with a view towards the pricing of futures derivatives. The second paper extends the LIBOR Market Model (LMM) of Miltersen et al. (1997) to make it compatible with the backward-looking benchmark. Both approaches allow for explicit modelling of the backward-looking benchmark, but both suffer from the the same drawbacks of the short-rate modelling and LMM frameworks, respectively. Neither approach allows for closed-form pricing of swaptions for higher dimensions of the state vector. In particular, even the simplest version of the LMM heavily relies on approximations for the pricing of swaptions and of futures. Our rational savings account models are inspired by the rational term structure models originally proposed in Flesaker and Hughston (1996) and more recently further developed in Akahori and Macrina (2012), Akahori et al. (2014), Macrina (2014), Nguyen and Seifried (2015), Crépey et al. (2016), Filipović et al. (2017), and Macrina and Mahomed (2018). Most of the rational term structures models allow for closed-form swaption pricing, but-as we will show-these models are not well-suited for modelling a backward-looking benchmark. We develop a different class of models which share similarities with the rational models framework but, instead of modelling the pricing kernel under the real-world measure $\mathbb{P}$, we model the savings account under the risk-neutral measure. Leaning on Döberlein and Schweizer (2001), we give conditions under which a rational savings account model may be expressed in terms of a short rate of interest process. As we shall show, rational savings account models are better suited for backward-looking interest rate benchmarks than the classical approach under the real-world probability measure. In particular, our framework allows for closed-form pricing of caplets, swaptions and futures, by which we mean that these prices can be calculated as one-dimensional numerical integrals. Hence, our model structure is tractable at a level comparable to the rational term structure models cited above.

1 One may expect that the Euro Overnight Index Average (EONIA), essentially a one-day EURIBOR, may in time become outdated as it is made superfluous by an established $€ \mathrm{STR}$. 
We begin with a general description of the model, and then we present the pricing formulas in a model driven by affine processes. We wrap up with concluding remarks and an outlook on future investigations.

\section{Derivatives on Backward-Looking Rates}

Let $\left(\Omega, \mathcal{F}, \mathbb{Q},\left(\mathcal{F}_{t}\right)_{t \in[0, \bar{T}]}\right)$ be a filtered probability space with $\bar{T}<\infty$. The bank account numeraire process $\left(B_{t}\right)_{t \geq 0}$ is associated with the risk-neutral measure denoted by $\mathbb{Q}$. It is customary to model the bank account numeraire by

$$
B_{t}=B_{0} \exp \left(\int_{0}^{t} r_{s} \mathrm{~d} s\right)
$$

where $\left(r_{t}\right)_{t \geq 0}$ is a specified instantaneous short rate. Instead, we shall work with a generalised notion of the bank account referred to as an implied savings account in Döberlein and Schweizer (2001). Thus we assume that $\left(B_{t}\right)_{t \geq 0}$ is a strictly positive process of finite variation, which generalises (1).

With LIBOR discontinued and superseded by an RFR benchmark, the rate-fixing and discounting are applied by using the same numeraire process $\left(B_{t}\right)_{t \geq 0}$. Hence no features typical of multi-curve interest rate systems emerge, and one is effectively back in a single-curve setting. The standard zero-coupon bond $(\mathrm{ZCB})$ has a price process $\left(P_{t T}\right)_{t \leq T}$ given by

$$
P_{t T}=B_{t} \mathbb{E}_{t}\left[B_{T}^{-1}\right]
$$

A European-style derivative with random payoff $X_{T}$ at time $T$ has at time $t \leq T$ a price given by

$$
S_{t}=\mathbb{E}_{t}\left[\frac{B_{t}}{B_{T}} X_{T}\right]
$$

Letting $\delta_{1 d}$ denote the day-count fraction of an overnight loan, it follows from standard interest rate theory that the discrete overnight rate is

$$
R_{1 d}(t):=\frac{1}{\delta_{1 d}}\left(1 / P_{t, t+\delta_{1 d}}-1\right)
$$

This is what ISDA refers to as the Risk-Free Rate (RFR), a benchmark rate that may correspond to SOFR, SONIA, €STR etc., in their respective currencies. The proposed LIBOR replacement is the following rate referred to as the backward-looking benchmark:

$$
\bar{R}(T, U)=\frac{1}{\delta}\left(\prod_{i=1}^{n_{\delta}}\left(1+\delta_{1 d} R_{1 d}\left(T_{i}\right)\right)-1\right), \quad \delta=U-T,
$$

where $T \leq T_{1}, \ldots, T_{n_{\delta}} \leq U$. A standard approximation applied in the literature, and in particular in Lyashenko and Mercurio (2019), is to replace discrete by continuous compounding and hence use

$$
\bar{R}(T, U) \approx R(T, U):=\frac{1}{\delta}\left(\frac{B_{U}}{B_{T}}-1\right)
$$

We will use this approximation throughout the paper. $^{2}$

We can compare this rate to a classical discrete forward-looking term rate

$$
F(T, U):=\left(1 / P_{T U}-1\right) / \delta
$$

2 After consecutive applications of the tower property one sees that, for $t \leq T \leq U, B_{t} \mathbb{E}_{t}\left[B_{T}^{-1} \bar{R}(t, T) \mid \mathcal{F}_{t}\right]=B_{t} \mathbb{E}_{t}\left[B_{T}^{-1} R(t, T)\right]$, and it thus follows that pricing linear derivatives, i.e., any derivative that is linear in the rate, is unaffected by the continuous compounding approximation in the case of $t \leq T \leq U$. 
again with tenor $\delta=U-T$, i.e., the rate on a loan that starts at time $T$ and has to be repaid at time $U$. The term rate $F(T, U)$ is based on the same curve as the backward-looking benchmark and can be thought of as LIBOR without the risk premia for credit and liquidity mentioned in the introduction.

In order to account for the risk premia, the actual LIBOR fallback used when converting existing LIBOR contracts will be $\bar{R}(T, U)+c$, where $c$ is determined by the historical relationship between LIBOR and $\bar{R}(T, U)$, see ISDA (2019). For ease of presentation, we shall ignore the spread, and we focus on the implications of changing to a backward-looking benchmark from a classical forward-looking rate. The first thing to notice is that $R(T, U)$ is $\mathcal{F}_{U}$-measurable while $F(T, U)$ is $\mathcal{F}_{T}$-measurable, where $T \leq U$. This change, while subtle, has substantial implications for the pricing of interest rate derivatives as we show in the next section.

\subsection{Swaplet}

Let us first consider the expectation of the discounted rate or what could be called a zero-strike swaplet, or one element in the floating leg of a swap. For $t \leq T \leq U$, we have

$$
\mathrm{SW}_{t}^{0}=\delta B_{t} \mathbb{E}_{t}\left[B_{U}^{-1} R(T, U)\right]=B_{t} \mathbb{E}_{t}\left[B_{T}^{-1}-B_{U}^{-1}\right]=\left(P_{t T}-P_{t U}\right)=\delta B_{t} \mathbb{E}_{t}\left[B_{U}^{-1} F(T, U)\right]
$$

The latter equality reveals that, for $t \leq T \leq U$, backward-looking rates are priced in the same way as the forward-looking equivalent, and by implication it follows that the valuation of linear derivatives remains essentially unaffected by the backward-looking nature of $R(T, U)$. However, for $t \in[T, U]$, one has instead

$$
\begin{aligned}
\mathrm{SW}_{t}^{0} & =\delta B_{t} \mathbb{E}_{t}\left[B_{U}^{-1} R(T, U)\right]=\frac{B_{t}}{B_{T}}-P_{t U} \\
& \neq \delta B_{t} \mathbb{E}_{t}\left[B_{U}^{-1} F(T, U)\right]=\frac{P_{t U}}{P_{T U}}-P_{t U}
\end{aligned}
$$

Thus when one moves to pricing the derivative after its initiation at $t>T$ the payoff is still undetermined as $R(T, U)$ is only measurable at time $U$. Relation (2) thus becomes explicitly dependent on the value of the bank account $B_{T}$, which is not the case in a classic forward-looking setting as given in the Expression (3).

\subsection{Forward Rate Agreement}

The forward rate agreement (FRA) based on a forward-looking benchmark rate, generates a cash flow

$$
\mathrm{FRA}_{T}=\frac{\delta(F(T, U)-K)}{1+\delta F(T, U)}
$$

at time $T$. In a single-curve setting, this can be considered a linear derivative by noting that for $t \leq T$

$$
\begin{aligned}
\mathrm{FRA}_{t} & =B_{t} \mathbb{E}_{t}\left[B_{T}^{-1} \frac{\delta(F(T, U)-K)}{1+\delta F(T, U)}\right]=B_{t} \mathbb{E}_{t}\left[B_{T}^{-1} P_{T U} \delta(F(T, U)-K)\right] \\
& =\delta B_{t} \mathbb{E}_{t}\left[B_{U}^{-1}(F(T, U)-K)\right]=S W_{t}^{0}-\delta K P_{t U}
\end{aligned}
$$

The price of the FRA thus becomes equivalent to the value of a contract with a cash flow $\delta(F(T, U)-K)$ at time $U$. It is not possible to readily substitute $F(T, U)$ with $R(T, U)$ in Equation (4), because the latter is not measurable at time $T$ (aka 'measurability problem'). This is likely to make transitioning FRA contracts from the forward- to the backward-looking benchmarks challenging as 
discussed in Henrard (2019). The closest backward-looking counterpart to the FRA is a swaplet paying $\delta(R(T, U)-K)$ at time $U$. This would be congruent with the standard FRA for $t \leq T$, since

$$
\mathrm{SW}_{t}^{K}=B_{t} \mathbb{E}_{t}\left[B_{U} \delta(R(T, U)-K)\right]=\mathrm{SW}_{t}^{0}-\delta K P_{t U}=\mathrm{FRA}_{t}
$$

However, for $t \in[T, U]$ we have

$$
\mathrm{SW}_{t}^{K}=\frac{B_{t}}{B_{T}}-(1+\delta K) P_{t U} \neq \mathrm{FRA}_{t}=\mathrm{FRA}_{T} \frac{1}{P_{T t}} .
$$

The fact that the payoff is random beyond the initiation point $T$ may appear inconsequential, but in practice it matters a great deal, for many risk management systems now have to be reconfigured to address correctly the randomness within the interval $[T, U]$. See again Henrard (2019) for further discussion.

\subsection{Caplet}

Similarly, a caplet written on the backward-looking rate would presumably pay $(R(T, U)-K)^{+}$ at time $U$. We can similarly price it for $\forall t \leq U$. Let $\tilde{K}=1+\delta K$, and we have

$$
\mathrm{CP}_{t}=\delta B_{t} \mathbb{E}_{t}\left[B_{U}^{-1}(R(T, U)-K)^{+}\right]=B_{t} \mathbb{E}_{t}\left[\left(B_{T}^{-1}-\tilde{K} B_{U}^{-1}\right)^{+}\right],
$$

which we can compare with the price of a standard caplet with payoff $\delta(F(T, U)-K)^{+}$at time $U$. For $t \leq T \leq U$ we have

$$
\delta B_{t} \mathbb{E}_{t}\left[B_{U}^{-1}(F(T, U)-K)^{+}\right]=B_{t} \mathbb{E}_{t}\left[B_{T}^{-1}\left(1-\tilde{K} P_{T U}\right)^{+}\right] \neq \mathrm{CP}_{t} .
$$

While the linear derivatives are priced the same in the backward- and forward-looking setting, at least for $t \leq T$, it is clear from the above equation that the caplets are not. In fact, as also shown in Lyashenko and Mercurio (2019) an application of Jensen's inequality to Equation (33) reveals that

$$
\begin{aligned}
\mathrm{CP}_{t} \geq B_{t} \mathbb{E}_{t}\left[\left(\mathbb{E}_{T}\left[B_{T}^{-1}-\tilde{K} B_{U}^{-1}\right]\right)^{+}\right] & \left.=B_{t} \mathbb{E}_{t}\left[\left(B_{T}^{-1}-\tilde{K} B_{T}^{-1} P_{T U}\right]\right)^{+}\right] \\
& =\delta B_{t} \mathbb{E}_{t}\left[B_{U}^{-1}(F(T, U)-K)^{+}\right]
\end{aligned}
$$

In other words a caplet written on the backward-looking benchmark is always worth more than or equal to its forward-looking counterpart.

\subsection{Swaps and swaptions}

An RFR swap can be defined as a contract that has a floating leg paying $\left\{R\left(T_{i-1}, T_{i}\right)\right\}_{\{i=a+1, \ldots, b\}}$ at times $T_{a+1}, \ldots, T_{b}$ and a fixed leg paying $K$ at the same dates. We can value the swap at time $t \leq T_{a}$ as

$$
V_{t}^{a, b}=B_{t} \mathbb{E}_{t}\left[\sum_{i=a+1}^{b} B_{T_{i}}^{-1} \delta\left(R\left(T_{i-1}, T_{i}\right)-K\right)\right]=P_{t T_{a}}-P_{t T_{b}}-\delta K \sum_{i=a+1}^{b} P_{t T_{i}} .
$$

We notice that, for $t \leq T_{a}, V_{t}^{a, b}$ is in fact equivalent to the value of a classical swap paying the discrete forward-looking rate $F\left(T_{i-1}, T_{i}\right)$ at time $T_{i}$ instead of $R\left(T_{i-1}, T_{i}\right)$ at time $T_{i}$. This is not the case for $t \in\left[T_{k-1}, T_{k}\right]$ for some $k \in(a+1, \ldots, b)$ where the value of future payments becomes

$$
V_{t}^{a, b}=B_{t} \mathbb{E}_{t}\left[\sum_{i=k}^{b} B_{T_{i}}^{-1} \delta\left(R\left(T_{i-1}, T_{i}\right)-K\right)\right]=\frac{B_{t}}{B_{T_{k}}}-P\left(t, T_{b}\right)-\delta K \sum_{i=k}^{b} P_{t T_{i}} .
$$


It again demonstrates that the underlying challenge with the backward-looking regime is that one gets explicit dependence on the savings account process once the valuation continues beyond the initiation date of the contract.

A swaption is an option to enter into a swap at time $T_{a}$, and we can of course price it for $t \leq T_{a}$ as

$$
\mathrm{SWP}_{t}=B_{t} \mathbb{E}_{t}\left[B_{T_{a}}^{-1}\left(V_{T_{a}}^{a, b}\right)^{+}\right] .
$$

Since the valuation of the swap is unaffected by whether or not the underlying rate is backwardor forward-looking if priced before the starting time $T_{a}$, it follows that swaption pricing is unaffected by the shift from forward to backward-looking rates.

\subsection{Considerations}

These preliminary examples illustrate that changing the benchmark from forward-looking to backward-looking rates will alter the pricing and risk management of linear and nonlinear derivatives. In particular (2) and (9) suggest that a modelling paradigm that provides an explicit expression for the bank account $\left(B_{t}\right)$ or more precisely its reciprocal $\left(B_{t}^{-1}\right)$ is advantageous. This is possible by using an affine short-rate model, via the relation $B_{t}=\exp \left(\int_{0}^{t} r_{s} \mathrm{~d} s\right)$, but, as is well-known, this choice does not in general lead to a closed-form solution for swaptions-approximations such as the one in Singleton and Umantsev (2002) have to be applied. Lyashenko and Mercurio (2019) extend the LMM framework to include explicit expressions for the bank account in the time interval $[T, U]$, but the framework suffers from the general tractability issues in the standard LMM, which involve non-trivial issues with Monte-Carlo simulation, and a lack of closed-form pricing for swaptions as well as futures.

A framework that prices swaptions in closed-form is one that results in discounted ZCB prices being linear in the spacial variable. The pricing kernel models by Flesaker and Hughston (1996) and Rutkowski (1997) are established examples thereof. This approach has been further studied and extended in Filipović et al. (2017), who demonstrate that it has excellent empirical properties. Its calibration properties in a multi-curve context are studied in Crépey et al. (2016). Such models of rational form could naturally be applied also to price swaptions on backward-looking rates. However, in the case of derivatives with payoffs requiring direct modelling of the savings account, it turns out that it is not as straightforward as that. Consider the well-known relation

$$
\pi_{t}=\left.\frac{\mathrm{d} \mathbb{P}}{\mathrm{d} \mathbb{Q}}\right|_{\mathcal{F}_{t}} B_{t}^{-1}
$$

where $\mathbb{Q}$ is the risk-neutral measure associated with the savings account (numeraire) process $\left(B_{t}\right)_{t \geq 0}$. If the aim is to obtain a rational form for the savings account process $\left(B_{t}\right)$, see Assumption 1 below, then modelling the pricing kernel $\left(\pi_{t}\right)$ directly may be a tedious path to take, which not necessarily will lead to the desired goal. What we are really interested in is an explicit expression for the process $\left(B_{t}\right)_{t \geq 0}$. Thus, we propose that the savings account process be of rational form, as postulated in Assumption 1 below. We will show that this allows for closed-form pricing under a risk-neutral measure of all derivatives written on a backward-looking interest rate benchmark studied in this section. For the theory underpinning the distinction between an (implied) savings account and a so-called classical savings account (one that may be written in terms of a short rate process), we refer to Döberlein and Schweizer (2001).

The discontinuation of interbank offered rate benchmarks has been described by some as the biggest experiment ever in financial markets. These are momentous times while market participants scramble to prepare for the transition to RFR benchmarks. The discontinuation of term-based benchmarks, such as LIBOR, reduces a multiple-curve interest rate system to a single-curve one, akin to the ones in place before the great financial crisis more than a decade ago. As discussed in Backwell et al. (2019), the lack of a term-rate system produces increased exposure to roll-over risk. As a 
consequence, one can expect a refinancing strategy to become costlier due to the increased roll-over frequency of debt that is rolled-over on an overnight basis. One ought to expect such effects to also impact any so-called 'LIBOR fallback' provisions and in particular the calculation of any adjustment (spread) to legacy contracts written on term-rates, see Mercurio (2018) and Henrard (2019) for example. The adoption of a consistent pricing and hedging approach that models and explains the nature of term rates and the impact of roll-over risk on borrowing strategies appears the right way forward. This would allow for a transparent treatment of term rates and overnight interest rate benchmarks so that a proposed transition to the latter has a chance to be appreciated in its entirety. A lack of term-based benchmark rates may produce a level of opacity in lending markets. Market makers may offer rates, which cannot be 'independently assessed' in the sense that a mark-to-market comparison to a FRA market is not going to be available. For first advancements on consistent approaches to term- and overnight interest rate benchmarks, we here refer to Alfeus et al. (2018), Backwell et al. (2019), and to an article by Macrina \& Mahomed (in preparation) with a focus on term risk in interest rate markets. We conclude our reflections by noting that, at the time of writing, there is no global consensus regarding the transition to RFRs. Depending on the jurisdiction, term-based benchmarks are to be superseded by RFRs (e.g., UK), term-based and RFR benchmarks shall coexist until both are accepted as market representatives (e.g., USA, Eurozone). Such ambiguities are perhaps a reflection of the differences between market structures, but also derive from the different natures of the benchmarks themselves that are being used.

\section{Rational savings account models}

In the case where one deals with backward-looking rates, we have now shown instances where the payoff itself will explicitly depend on the savings account. It is therefore a clear advantage to directly model the process $\left(B_{t}\right)_{t \geq 0}$. Building on Döberlein and Schweizer (2001), we make the following assumption throughout this paper:

Assumption 1. The savings account model $\left(B_{t}\right)_{t \geq 0}$ is given by

$$
B_{t}:=\frac{1}{a(t)+b(t) Y_{t}}
$$

for all $t \geq 0$, and where $\left(Y_{t}\right)_{t \geq 0}$ is a stochastic, nonnegative process of finite variation. The function $a(t)$ is deterministic, strictly positive, of bounded variation while $b(t)$ is deterministic, nonnegative and of bounded variation. It is assumed they satisfy the initial condition,

$$
a(0)+b(0) Y_{0}=1 .
$$

The financial or economic interpretation of the savings account is no different from that of a classical bank account. The savings account $\left(B_{t}\right)_{t \geq 0}$ discounts/accrues cash flows, and, as the name suggests, quantifies the savings made over a period of time. It is also the numeraire associated with an equivalent risk-neutral measure. The difference here is that the savings account model, given by Definition (16), is more general than bank account processes defined in terms of a short-rate model. The rational form of Relation (16) allows for an increased level of tractability, as we will see next, when deriving the price dynamics of interest rate derivatives under a risk-neutral measure $\mathbb{Q}$.

Lemma 1. The savings account $\left(B_{t}\right)_{t \geq 0}$, defined by Equation (16), is a strictly positive and finite-variation process.

Proof. Let the sample path for a given $\omega \in \Omega$ be $h: t \rightarrow Y_{t}(\omega)$ and define the mapping $g: t \rightarrow$ $a(t)+b(t) h(t)$. The functions $a, b$ and $h$ have bounded variation. It follows from the chain rule of bounded variation, see Ambrosio and Dal Masio (1990), that the product (and trivially the sum) of two 
functions of bounded variation are of bounded variation. Hence, the mapping $g$ has bounded variation. Let $f: x \rightarrow 1 / x$. Then the savings account is given by the composite function $B_{t}(\omega)=f \circ g(t, \omega)$. The function $f$ is Lipschitz-continuous and strictly positive on $\mathbb{R}_{>0}$. By another application of the chain rule result, it turns out that the composite function $B_{t}(\omega)$ is of bounded variation for all $\omega \in \Omega$ and thus that the stochastic process $\left(B_{t}\right)_{t \geq 0}$ is strictly positive and has finite variation for all $t \geq 0$.

Lemma 1 establishes that the specification in Equation (16) is a savings account in the sense of Döberlein and Schweizer (2001). The probability measure $\mathbb{Q}$, associated with the numeraire process $\left(B_{t}\right)_{t \geq 0}$, is hence a risk-neutral measure.

Remark 1. If any of the conditions underpinning Lemma 1 are relaxed, resulting in $\left(B_{t}\right)_{t \geq 0}$ being a positive semi-martingale that is not of finite variation, then $\left(B_{t}\right)_{t \geq}$ is no longer a savings account. It would however still qualify as a numeraire and its associated measure would still be a martingale measure, though not a risk-neutral measure. This means that the true savings account process would no longer be directly specified, thus making the modelling advantage of our approach void.

We are now in the position to write down the price process of a discount bond:

$$
P_{t T}=B_{t} \mathbb{E}_{t}\left[B_{T}^{-1}\right]=\frac{a(T)+b(T) \mathbb{E}_{t}\left[Y_{T}\right]}{a(t)+b(t) Y_{t}},
$$

where we recall that the conditional expectation is taken with respect to the risk-neutral measure $\mathbb{Q}$. Assuming that the bond price function $P(t, T)$ is differentiable in $T$, the instantaneous bond return process $\left(\eta_{t}\right)_{t \geq 0}$ has the form

$$
\eta_{t}=-\left.\partial_{T} \ln \left(P_{t T}\right)\right|_{T=t}=-\frac{\dot{a}(t)+\left.\dot{b}(t) \mathbb{E}_{t}\left[Y_{T}\right]\right|_{T=t}+\left.b(t)\left(\partial_{T} \mathbb{E}_{t}\left[Y_{T}\right]\right)\right|_{T=t}}{a(t)+\left.b(t) \mathbb{E}_{t}\left[Y_{T}\right]\right|_{T=t}},
$$

where the dot-notation stands for differentiation with respect to $t$. It is worth emphasising that the instantaneous bond return may not be given by a short rate, as used in Equation (1), in general. While $\left(Y_{t}\right)_{t \geq 0}$ is of finite variation, $\left(\mathbb{E}_{t}\left[Y_{T}\right]\right)_{t \in[0, T]}$ is not of finite variation, in general. It follows that the ZCB price process and the instantaneous bond return process may also be of infinite variation. The above expression also reveals that, even in the case of a strictly increasing process $\left(Y_{t}\right)_{t \geq 0}$, the support of $\left(\eta_{t}\right)_{t \geq 0}$ is not limited to a positive domain. Its sign is controlled by the time-derivatives $\dot{a}(t)$ and $\dot{b}(t)$, which may have either sign, and the model can produce positive as well as negative rates. Furthermore, the Form (17) of the discount bond allows for the calibration of the price model to the discount bond initial term-structure $\left(P_{0 t}\right)_{0 \leq t \leq T}$. We have

$$
P_{0 t}=a(t)+b(t) \mathbb{E}\left[Y_{t}\right],
$$

where $0 \leq t \leq T$. Solving for $a(t)$ and inserting the result into Equation (17), one obtains

$$
P_{t T}=\frac{P_{0 T}+b(T)\left(\mathbb{E}_{t}\left[Y_{T}\right]-\mathbb{E}\left[Y_{T}\right]\right)}{P_{0 t}+b(t)\left(Y_{t}-\mathbb{E}\left[Y_{t}\right]\right)} .
$$

Similar to an HJM-style model, the deterministic function $a(t)_{0 \leq t \leq T}$ may be automatically calibrated to the initial term structure while $b(t)_{0 \leq t \leq T}$ and the specific parameters related to the process $\left(Y_{t}\right)_{t \geq 0}$ can be used to calibrate to non-linear derivatives.

Assumption 2. The stochastic process $\left(Y_{t}\right)_{t \geq 0}$ is taken to be

$$
Y_{t}=\int_{0}^{t}\left(w \cdot Z_{s}\right) \mathrm{d} s, \quad t \geq 0,
$$


where $w \in \mathbb{R}_{\geq 0}^{d-1}$ and $\left(Z_{t}\right)_{t \geq 0}$ is an affine process, as characterised in the appendix, defined on $\mathbb{R}_{\geq 0}^{d-1}$ and such that $w \cdot Z_{0}>0$.

Remark 2. In line with Proposition 12 in Döberlein and Schweizer (2001), for a process $\left(Y_{t}\right)_{0 \leq t}$ of the form (21)—not necessarily defined in terms of an affine process—such that $\mathbb{E}\left[w \cdot Z_{t}\right]<\infty$ for all $t \geq 0$, it follows that the dynamics of the savings account, given by Relation (16), may be written as $d B_{t}=r_{t} B_{t} \mathrm{~d} t$, where

$$
r_{t}:=-\frac{\dot{a}(t)+\dot{b}(t) Y_{t}+b(t) w \cdot Z_{t}}{a(t)+b(t) Y_{t}}
$$

is the short rate of interest. This is obtained by applying Ito's quotient rule. It then also follows that the instantaneous return, with Form (18), on the discount bond satisfies $\eta_{t}=r_{t}, 0 \leq t \leq T$.

It follows from standard theory that $\left(Y_{t}\right)_{t \geq 0}$ is strictly positive and of finite variation. Theorem 4.1 in Keller-Ressel (2008) states that if one defines

$$
X_{t}:=\left(Y_{t}, Z_{t}\right), \quad \forall t \geq 0,
$$

then $\left(X_{t}\right)_{t \geq 0}$ is affine in $\mathbb{R}_{\geq 0}^{d}$. By definition of an affine process, there exist functions $\phi:[0, \bar{T}] \times \mathbb{C}^{d} \rightarrow \mathbb{C}$ and $\psi:[0, \bar{T}] \times \mathbb{C}^{d} \rightarrow \mathbb{C}^{d}$ such that

$$
\mathbb{E}_{t}\left[\mathrm{e}^{u \cdot X_{T}}\right]=\exp \left(\phi_{T-t}(u)+\psi_{T-t}(u) \cdot X_{t}\right)
$$

for all $t \in[0, T]$. Here, $u \in S(\mathcal{I})$ where

$$
\mathcal{I}:=\left\{u \in \mathbb{R}^{d}: \sup _{t \in[0, \bar{T}]} \mathbb{E}\left[\mathrm{e}^{u \cdot X_{t}}\right]<\infty\right\}
$$

and where $S(\mathcal{I}):=\left\{z \in \mathbb{C}^{d}: \Re z \in \mathcal{I}\right\}$ is the the strip of existence. We assume that $0 \in \operatorname{int}(\mathcal{I})$ where int denotes the interior of the set $\mathcal{I}$. Furthermore, we will often need the derivatives with respect to the vector $u=\left[u_{1}, \ldots, u_{d}\right]^{\top}$, and so

$$
\begin{aligned}
& \frac{\partial \phi_{T-t}(u)}{\partial u}=\left[\frac{\partial \psi_{T-t}(u)}{\partial u_{1}}, \ldots, \frac{\partial \psi_{T-t}(u)}{\partial u_{d}}\right]^{\top}, \\
& \frac{\partial \psi_{T-t}(u)}{\partial u}=\left[\frac{\partial \psi_{T-t}(u)}{\partial u_{1}}, \ldots, \frac{\partial \psi_{T-t}(u)}{\partial u_{d}}\right]=\left[\begin{array}{ccc}
\frac{\partial \psi_{T-t}^{1}(u)}{\partial u_{1}} & \ldots & \frac{\partial \psi_{T-t}^{1}(u)}{\partial u_{d}} \\
\vdots & \ddots & \vdots \\
\frac{\partial \psi_{T-t}^{d}(u)}{\partial u_{1}} & \ldots & \frac{\partial \psi_{T-t}^{d}(u)}{\partial u_{d}}
\end{array}\right] .
\end{aligned}
$$

Next, we derive the pricing formulas of the main fixed-income derivative instruments under Assumptions 1 and 2.

\subsection{Linear derivatives}

We next make use of the savings account process introduced in Assumption 1, and in particular consider the setting in Assumption 2, to derive the no-arbitrage price process of linear derivatives written on a backward-looking benchmark. In the following proposition, we begin with a discount bond. 
Proposition 1. The discount bond price process is given by

$$
P_{t T}=\frac{a(T)+b(T)\left(\left.\frac{\partial \phi_{T-t}(u)}{\partial u_{1}}\right|_{u=0}+\left.\frac{\partial \psi_{T-t}(u)}{\partial u_{1}}\right|_{u=0} \cdot X_{t}\right)}{a(t)+b(t) Y_{t}}=\frac{\tilde{a}(t, T)+\tilde{b}(t, T) \cdot X_{t}}{a(t)+b(t) Y_{t}}
$$

where $\tilde{a}(t, T)=a(T)+\left.b(T) \frac{\partial \phi_{T-t}(u)}{\partial u_{1}}\right|_{u=0}$ and $\tilde{b}(t, T)=\left.b(T) \frac{\partial \psi_{T-t}(u)}{\partial u_{1}}\right|_{u=0}$.

Proof. We apply Lemma A4 to obtain

$$
\begin{aligned}
\mathbb{E}_{t}\left[Y_{T}\right]=\mathbb{E}_{t}\left[e_{1} \cdot X_{T}\right] & =e_{1} \cdot\left(\left.\frac{\partial \phi_{T-t}(u)}{\partial u}\right|_{u=0}+\left(\left.\frac{\partial \psi_{T-t}(u)}{\partial u}\right|_{u=0}\right)^{\top} X_{t}\right) \\
& =\left(\left.\frac{\partial \phi_{T-t}(u)}{\partial u_{1}}\right|_{u=0}+\left.\frac{\partial \psi_{T-t}(u)}{\partial u_{1}}\right|_{u=0} \cdot X_{t}\right),
\end{aligned}
$$

where $e_{1}$ is a $d$-dimensional unit-vector with one in the first entry and zero elsewhere. Inserting the above in Equation (17) yields the Equality (27).

\subsection{Caplets and Swaptions}

Proposition 2. The price of a caplet paying $(R(T, U)-K)^{+}$at time $U$ for any $t \leq U$ is,

$$
\begin{aligned}
C P_{t} & =\delta B_{t} \mathbb{E}_{t}\left[B_{U}^{-1}(R(T, U)-K)^{+}\right]=\delta B_{t} \mathbb{E}_{t}\left[\left(c_{0}+c_{1} \cdot X_{T}+c_{2} \cdot X_{U}\right)^{+}\right] \\
& =\frac{\delta B_{t}}{\pi} \int_{0}^{\infty} \Re\left[\frac{\hat{q}(R+i v)}{(R+i v)^{2}}\right] \mathrm{d} v,
\end{aligned}
$$

where $\delta=U-T, \tilde{K}=1+\delta K, c_{1}=e_{1} B_{T}, c_{2}=e_{1} \tilde{K} B_{U}$ and

$$
\begin{aligned}
\hat{q}(k): & =\mathbb{E}_{t}\left[\mathrm{e}^{k\left(c_{0}+c_{1} \cdot X_{T}+c_{2} \cdot X_{U}\right.}\right] \\
& =\left\{\begin{array}{ll}
\mathrm{e}^{k c_{0}+\phi_{T-U}\left(c_{2}\right)+\phi_{T-t}\left(c_{1}+\psi_{T-U}\left(c_{2}\right)\right)+\psi_{T-t}\left(c_{1}+\psi_{T-t}\left(c_{2}\right)\right) \cdot X_{t}}, & \forall t \leq T<U, \\
\mathrm{e}^{k c_{0}+c_{1} \cdot X_{T}+\phi_{U-t}\left(k c_{2}\right)+\psi_{U-t}\left(k c_{2}\right) \cdot X_{t},} & \forall t \in[T, U]
\end{array},\right.
\end{aligned}
$$

for any $k \in \mathbb{C}$ such that $k c_{1}, k c_{2} \in S(\mathcal{I})$ and where $R$ is any real constant such that $\hat{q}(R)<\infty$.

Proof. Relation (30) follows by direct insertion of the definition of $\left(B_{t}\right)$ and $R(T, U)$ and from rewriting the expression as a function of $X_{T}$ and $X_{U}$. Result (31) follows directly from Theorem 4 in Filipović et al. (2017), and Equation (33) follows from Lemma A5 in Appendix B .

The following proposition shows that a closed-form formula for swaptions similar to those derived in, e.g., Flesaker and Hughston (1996) and other works on rational models, can be derived in our setup.

Proposition 3. The price of a swaption is given as

$$
\begin{aligned}
S W P_{t} & =B_{t} \mathbb{E}_{t}\left[B\left(T_{a}\right)^{-1}\left(V_{T_{a}}^{a, b}\right)^{+}\right]=B_{t} \mathbb{E}_{t}\left[\left(\tilde{a}^{a, b}+\tilde{b}^{a, b} \cdot X_{T_{a}}\right)^{+}\right] \\
& =\frac{B_{t}}{\pi} \int_{0}^{\infty} \Re\left[\frac{\hat{q}(R+i v)}{(R+i v)^{2}}\right] \mathrm{d} v,
\end{aligned}
$$

where $\tilde{a}^{a, b}=\tilde{a}\left(t, T_{a}\right)-\tilde{a}\left(t, T_{b}\right)-\delta K \sum_{i=a+1}^{b} \tilde{a}\left(t, T_{i}\right)$ and $\tilde{b}^{a, b}=\tilde{b}\left(t, T_{a}\right)-\tilde{b}\left(t, T_{b}\right)-\delta K \sum_{i=a+1}^{b} \tilde{b}\left(t, T_{i}\right)$ and we have

$$
\hat{q}(k)=\mathbb{E}_{t}\left[\mathrm{e}^{k\left(\tilde{a}^{a, b}+\tilde{b}^{a, b} \cdot X_{t}\right)}\right]=\mathrm{e}^{k \tilde{a}^{a, b}} \mathrm{e}^{\left.\phi_{T_{a}-t}\left(k \tilde{b}^{a, b}\right)+\psi_{T_{a}-t}\left(k \tilde{b}^{a, b}\right) \cdot X_{t}\right)}
$$


For all $k \in \mathbb{C}$ such that $k \tilde{b}^{a, b} \in S(\mathcal{I}) . R>0$ is any real constant such that $\hat{q}(R)<\infty$.

Proof. Relation (34) follows by direct insertion of Expression (27) into Relation (13) and from rewriting the expression as a function of $X_{T_{a}}$. Equation (35) follows by an application of Theorem 4 in Filipović et al. (2017).

\subsection{Futures}

Finally, we consider the contract of a future. A standard result, presented in, e.g., Hunt and Kennedy (2004), is that the price of a future contract with a random payoff $X$, measurable at time $U$, is priced as the risk-neutral expectation thereof, without discounting, that is, $\mathbb{E}_{t}[X]$. The convention for three-month SOFR/SONIA futures is that their payoff is given by the difference between a notional and the discretely compounded backward-looking rate. Assuming a unit notional, the payoff is $1-(1+\delta \bar{R}(T, U))$, where $\delta=U-T \approx$ three months. As in Mercurio (2018), we use $\mathbb{E}_{t}[\bar{R}(T, U)] \approx$ $\mathbb{E}_{t}[R(T, U)]$ and define the futures rate for a three-month contract by

$$
f^{3 m}(t)=\mathbb{E}_{t}[R(T, U)]
$$

In a LIBOR market model, this expectation cannot be solved in closed-form; an approximation is developed in Lyashenko and Mercurio (2019). In the following proposition, we show that in our setting no approximation is needed.

Proposition 4. The futures rate for a three-month SOFR futures contract expiring at time $U$ with $\delta=U-T \approx$ three months is given by

$$
f^{3 m}(t, U)=\mathbb{E}_{t}[R(T, U)]=\frac{1}{\delta}\left(\int_{0}^{\infty} \mathrm{e}^{c_{2}(s)+\phi_{T-t}\left(v_{2}(s)\right)+\psi_{T-t}\left(v_{2}(s)\right) \cdot X_{t}}\left(\bar{a}(s)+\bar{b}(s) \cdot X_{t}\right) \mathrm{d} s-1\right),
$$

where $\bar{a}(s)=a(T)+\left.b(T) \frac{\partial \phi_{T-t}(u)}{\partial u_{1}}\right|_{u=v_{2}(s)}$ and $\bar{b}(s)=\left.b(T) \frac{\partial \psi_{T-t}(u)}{\partial u_{1}}\right|_{u=v_{2}(s)}$. Moreover,

$$
v_{2}(s):=\psi_{U-T}\left(-s e_{1} B_{U}\right), \quad c_{2}(s):=-s a(U)+\phi_{U-T}\left(-s e_{1} B_{U}\right) .
$$

Proof. From Lemma A2 (see Appendix A) we obtain

$$
\begin{aligned}
\mathbb{E}_{T}\left[B_{U}\right] & =\mathbb{E}_{T}\left[\left(a(U)+b(U) Y_{U}\right)^{-1}\right]=\int_{0}^{\infty} \mathbb{E}_{T}\left[\mathrm{e}^{-s\left(a(U)+b(U) Y_{U}\right)}\right] \mathrm{d} s \\
& =\int_{0}^{\infty} \exp \left(-s a(U)+\phi_{U-T}\left(-s e_{1} b(U)\right)+\psi_{U-T}\left(-s e_{1} b(U)\right) \cdot X_{T}\right) \mathrm{d} s
\end{aligned}
$$

Then,

$$
\begin{aligned}
& \mathbb{E}_{t}\left[\frac{B_{U}}{B_{T}}\right]=\mathbb{E}_{t}\left[\frac{1}{B_{T}} \mathbb{E}_{T}\left[B_{U}\right]\right] \\
& =\mathbb{E}_{t}\left[\left(a(T)+b(T) Y_{T}\right) \int_{0}^{\infty} \mathrm{e}^{-s a(U)+\phi U-T\left(-s e_{1} b(U)\right)+\psi_{U-T}\left(-s e_{1} b(U)\right) \cdot X_{T}} \mathrm{~d} s\right] \\
& =\int_{0}^{\infty} \mathrm{e}^{-s a(U)+\phi\left(-T-T\left(-s e_{1} b(U)\right)\right.} \mathbb{E}_{t}\left[\left(a(T)+b(T) e_{1} \cdot X_{T}\right) \mathrm{e}^{\psi_{U-T}\left(-s e_{1} b(U)\right) \cdot X_{T}}\right] \mathrm{d} s \\
& =\int_{0}^{\infty} \mathrm{e}^{c_{2}(s)+\phi_{T-t}\left(v_{2}(s)\right)+\psi_{T-t}\left(v_{2}(s)\right) \cdot X_{t}} \\
& \quad \times\left(a(T)+b(T) e_{1} \cdot\left[\left.\frac{\partial \phi_{T-t}(u)}{\partial u}\right|_{u=v_{2}(s)}+\left(\left.\frac{\partial \psi_{T-t}(u)}{\partial u}\right|_{u=v_{2}(s)}\right)^{\top} X_{t}\right]\right) \mathrm{d} s,
\end{aligned}
$$


from which Equation (38) follows. In the third equality, Fubini's theorem is applied. This is valid subject to $\psi_{U-T}\left(-s e_{1} b(U)\right) \in \mathcal{I}$ for all $s \geq 0$. This condition holds because $-s e_{1} b(U) \in \mathcal{I}$ for all $s \geq 0$.

The convention for one-month SOFR futures is that their payoff is an arithmetic average rate instead of a geometric compounded rate. Following Mercurio (2018), we define the rate of one-month futures expiring at time $U$, where $1 m \approx \delta=U-T$, by

$$
f^{1 m}(t, U)=\mathbb{E}_{t}\left[R^{1 m}(T, U)\right]
$$

where $R^{1 m}(T, U)$ is the arithmetic average of the overnight rate over one month. Then,

$$
R^{1 m}(T, U)=\frac{1}{\delta} \sum_{i=1}^{n_{1 M}} R_{1 d}\left(T_{i}\right) \delta_{1 d}, \quad R_{1 d}(t)=\frac{1}{\delta_{1 d}}\left(\frac{1}{P\left(t, t+\delta_{1 d}\right)}-1\right) .
$$

Here, $T=T_{1}<\cdots<T_{n_{1 m}+1}=U$ spans the course of one month. This case is not covered in the LMM context of Lyashenko and Mercurio (2019), and it is not clear how one would proceed without further approximations. It is investigated in a short rate model in Mercurio (2018), where the arithmetic average is approximated by an integral over the short rate of interest. This approximation simplifies the expression for Solution (46) in an affine short rate model, but it is in fact not necessary-even for the short rate model they present. As shown below, tractability depends on obtaining an analytical result for the expression $\mathbb{E}_{t}\left[1 / P_{T U}\right]$, which exists by definition in an exponential affine short rate model. In our savings account setting the resulting expression for the conditional expectation is a little more involved, but nevertheless it only amounts to performing a one-dimensional integration.

Lemma 2. For all $t \in[T, U)$ we have

$$
\mathbb{E}_{t}\left[1 / P_{T U}\right]=\int_{0}^{\infty}\left[a(T) q_{1}(-s)+b(T) q_{2}(-s)\right] \mathrm{d} s,
$$

where

$$
\begin{aligned}
q_{1}(s) & =\mathbb{E}_{t}\left[\mathrm{e}^{s\left[\tilde{a}(T, U)+\tilde{b}(T, U) \cdot X_{T}\right]}\right]=\mathrm{e}^{s \tilde{a}(T, U)+\phi_{T-t}(s \tilde{b}(T, U))+\psi_{T-t}\left(s \tilde{b}(T, U) \cdot X_{t}\right.}, \\
q_{2}(s) & =\mathbb{E}_{t}\left[Y_{T} \mathrm{e}^{s\left[\tilde{a}(T, U)+\tilde{b}(T, U) \cdot X_{T}\right]}\right] \\
& =\mathrm{e}^{s \tilde{a}(T, U)+\phi_{T-t}(s \tilde{b}(T, U))+\psi_{T-t}(\tilde{s} \tilde{b}(T, U)) \cdot X_{t}}\left[\left.\frac{\partial \phi_{T-t}(u)}{\partial u_{1}}\right|_{u=s \tilde{b}(T, U)}+\left(\left.\frac{\partial \psi_{T-t}(u)}{\partial u_{1}}\right|_{u=s \tilde{b}(T, U)}\right) \cdot X_{t}\right] .
\end{aligned}
$$

\section{Proof.}

$$
\begin{aligned}
\mathbb{E}_{t}\left[1 / P_{T U}\right] & =\mathbb{E}_{t}\left[\frac{a(T)+b(T) Y_{T}}{\tilde{a}(T, U)+\tilde{b}(T, U) \cdot X_{t}}\right] \\
& =a(T) \mathbb{E}_{t}\left[\frac{1}{\tilde{a}(T, U)+\tilde{b}(T, U) \cdot X_{T}}\right]+b(T) \mathbb{E}_{t}\left[Y_{T} \frac{1}{\tilde{a}(T, U)+\tilde{b}(T, U) \cdot X_{T}}\right] .
\end{aligned}
$$

Applying Lemma A2 to the first term yields

$$
\begin{aligned}
\mathbb{E}_{t}\left[\left(\tilde{a}(T, U)+\tilde{b}(T, U) \cdot X_{T}\right)^{-1}\right] & =\int_{0}^{\infty} \mathbb{E}_{t}\left[\mathrm{e}^{\left.-s\left[\tilde{a}(T, U)+\tilde{b}(T, U) \cdot X_{T}\right]\right]} \mathrm{d} s\right. \\
& =\int_{0}^{\infty} \mathrm{e}^{-s \tilde{a}(T, U)+\phi_{T-t}(-s \tilde{b}(T, U))+\psi_{T-t}(-s \tilde{b}(T, U)) \cdot X_{t}} \mathrm{~d} s
\end{aligned}
$$


Similarly, by applying Lemmas A3 and A4 to the second term gives

$$
\begin{aligned}
& \mathbb{E}_{t}\left[Y_{T}\left(\tilde{a}(T, U)+\tilde{b}(T, U) \cdot X_{T}\right)^{-1}\right]=\int_{0}^{\infty} \mathbb{E}_{t}\left[Y_{T} \mathrm{e}^{-s\left(\tilde{a}(T, U)+\tilde{b}(T, U) \cdot X_{T}\right)}\right] \mathrm{d} s \\
& =\int_{0}^{\infty}\left[\mathrm{e}^{s \tilde{a}(T, U)+\phi_{T-t}(s \tilde{b}(T, U))+\psi_{T-t}(s \tilde{b}(T, U)) \cdot X_{t}}\left[\left.\frac{\partial \phi_{T-t}(u)}{\partial u_{1}}\right|_{u=s \tilde{b}(T, U)}+\left(\left.\frac{\partial \psi_{T-t}(u)}{\partial u_{1}}\right|_{u=s \tilde{b}(T, U)}\right) \cdot X_{t}\right]\right] \mathrm{d} s .
\end{aligned}
$$

With this result at hand, we can now express the one-month futures rate:

\section{Proposition 5.}

$$
f^{1 m}(t, U)=\mathbb{E}_{t}\left[R^{1 M}(T, U)\right]=\frac{1}{\delta} \int_{0}^{\infty} \sum_{i=1}^{n_{1 M}}\left(a\left(T_{i}\right) q_{1}\left(t, T_{i}, s\right)+b\left(T_{i}\right) q_{2}\left(t, T_{i}, s\right)\right) \mathrm{d} s-\frac{n_{1 m}}{\delta},
$$

where

$$
\begin{aligned}
q_{1}(t, T, s) & \left.=\mathbb{E}_{t}\left[\mathrm{e}^{s\left[\tilde{a}\left(T, T+\delta_{1 d}\right)+\tilde{b}\left(T, T+\delta_{1 d}\right) \cdot X_{T}\right]}\right]=\mathrm{e}^{s \tilde{a}(T, U)+\phi_{T-t}\left(s \tilde{b}\left(T, T+\delta_{1 d}\right)\right)+\psi_{T-t}\left(s \tilde{b}\left(T, T+\delta_{1 d}\right) \cdot X_{t}\right.}\right] \\
q_{2}(t, T, s)= & \mathbb{E}_{t}\left[Y_{T} \mathrm{e}^{s\left[\tilde{a}\left(T, T+\delta_{1 d}\right)+\tilde{b}\left(T, T+\delta_{1 d}\right) \cdot X_{T}\right]}\right] \\
= & \mathrm{e}^{s \tilde{a}\left(T, T+\delta_{1 d}\right)+\phi_{T-t}\left(s \tilde{b}\left(T, T+\delta_{1 d}\right)\right)+\psi_{T-t}\left(s \tilde{b}\left(T, T+\delta_{1 d}\right)\right) \cdot X_{t}} \\
& \times\left[\left.\frac{\partial \phi_{T-t}(u)}{\partial u_{1}}\right|_{u=s \tilde{b}\left(T, T+\delta_{1 d}\right)}+\left(\left.\frac{\partial \psi_{T-t}(u)}{\partial u_{1}}\right|_{u=s \tilde{b}\left(T, T+\delta_{1 d}\right)}\right) \cdot X_{t}\right] .
\end{aligned}
$$

Proof. A direct application of Lemma 2 in Relation (46) confirms the result.

Example: Multidimensional CIR Process

We conclude with a sketch of an example. Assume $X_{t}=\left(Y_{t}, Z_{t}\right)=\left(\int_{0}^{t} w \cdot Z_{s} d s, Z_{t}\right)$ where $Z_{t}=\left[Z_{t}^{1}, \ldots, Z_{t}^{d-1}\right]^{\top}$ and where $Z_{t}^{i}$ is given by

$$
\mathrm{d} Z_{t}^{i}=\kappa_{i}\left(\theta_{i}-Z_{t}^{i}\right) \mathrm{d} t+\sigma_{i} \sqrt{Z_{t}^{i}} \mathrm{~d} W_{t}^{i}, \quad Z_{0}^{i}>0, \quad \forall i=1, \ldots, d-1,
$$

and $\kappa_{i}, \theta_{i}, \sigma_{i}>0$ and $\left(W_{t}^{i}\right)$ is standard Brownian motion independent of $\left(W_{t}^{j}\right)$ for $i \neq j$. The conditional moment generating function $\mathbb{E}_{t}\left[\mathrm{e}^{X_{T}}\right]=\mathrm{e}^{\phi_{T-t}(u)+\psi_{T-t}(u) \cdot X_{t}}$ for $\left(X_{t}\right)_{t \geq 0}$ defined above is found by first noticing that

$$
\mathrm{e}^{u \cdot X_{t}}=\prod_{i=1}^{d-1} \exp \left(u_{1} w_{i} \int_{0}^{t} Z_{s}^{i} d s+u_{i+1} Z_{t}^{i}\right)
$$

Using the independence property of the processes $\left(Z_{t}^{i}\right)_{t \geq 0}$ and applying Lemma A6 gives

$$
\begin{aligned}
\phi_{T-t}(u) & =\sum_{i=1}^{d-1} A_{T-t}^{i}\left(w_{i} u_{1}, u_{i+1}\right), \quad \psi_{T-t}(u)=\left[u_{1}, B_{T-t}^{1}\left(w_{1} u_{1}, u_{2}\right), \ldots, B_{T-t}^{d-1}\left(w_{d-1} u_{1}, u_{d}\right)\right]^{\top}, \\
A_{s}^{i}\left(v_{1}, v_{2}\right) & =\frac{2 \kappa_{i} \theta_{i}}{\sigma_{i}^{2}} \ln \left(\frac{2 \gamma_{i} \mathrm{e}^{\left(\gamma_{i}+\kappa_{i}\right) s / 2}}{\left(\kappa_{i}-v_{2} \sigma_{i}^{2}\right)\left(\mathrm{e}^{\gamma_{i} s}-1\right)+\gamma_{i}\left(\mathrm{e}^{\gamma_{i} s}+1\right)}\right), \\
B_{s}^{i}\left(v_{1}, v_{2}\right) & =\frac{v_{2}\left(\kappa_{i}+\gamma_{i}+\mathrm{e}^{\gamma_{i} s}\left(\gamma_{i}-\kappa_{i}\right)\right)+2 v_{1}\left(\mathrm{e}^{\gamma_{i} s}-1\right)}{\left(\kappa_{i}-v_{2} \sigma_{i}^{2}\right)\left(\mathrm{e}^{\gamma_{i} s}-1\right)+\gamma_{i}\left(\mathrm{e}^{\gamma_{i} s}+1\right)}, \\
\gamma_{i} & =\sqrt{\kappa_{i}^{2}-2 \sigma_{i}^{2} v_{1}} .
\end{aligned}
$$


It follows from Lemma A6 that the result holds for the set $\left\{u \in \mathbb{R}^{d} \mid u_{1} w_{i}<\frac{\kappa_{i}^{2}}{2 \sigma_{i}^{2}} \& u_{i+1}<\right.$ $\left.\kappa_{i} / \sigma_{i}^{2}, \quad \forall i=1, \ldots d-1\right\}$. The derivatives needed in the pricing equations can be calculated as follows:

$$
\begin{aligned}
\frac{\partial \phi_{t}(u)}{\partial u_{1}} & =\sum_{i=1}^{d-1} \frac{\partial A_{T-t}^{i}\left(w_{i} u_{1}, u_{i+1}\right)}{\partial u_{1}}, \\
\frac{\partial \psi_{t}(u)}{\partial u_{1}} & =\left[1, \frac{\partial B_{T-t}^{1}\left(w_{1} u_{1}, u_{2}\right)}{\partial u_{1}}, \ldots, \frac{\partial B_{T-t}^{d-1}\left(w_{d-1} u_{1}, u_{d}\right)}{\partial u_{1}}\right]^{\top}
\end{aligned}
$$

The explicit expressions are lengthy and tedious to calculate by hand, but can be obtained with ease by using a software such as Mathematica offering symbolic calculations. In Appendices A and B, we include some results that assist with the mathematical developments in this section.

\section{Conclusions and Outlook}

In this paper we develop a new class of rational term structure models that is tailored to the new backward-looking interest rate benchmarks, which are meant to supersede the forward-looking LIBOR. Currently, only the three-month SOFR and SONIA futures can be said to be traded on the new overnight benchmarks, and trading volumes are still rather limited, but nevertheless growing as the LIBOR discontinuation deadline nears. As trade volumes in SOFR/SONIA-based contracts rise, we expect that swaps, caps and swaptions enter the market in a fashion similar to what is described in this paper. A maturing market will generate the needed data also to perform empirical investigations on the validity of the proposed models. At present, not even linear derivatives are traded liquidly, meaning that an initial term structure $\left(P_{0 t}\right)_{t \geq 0}$ cannot be implied in a straightforward way. An implied initial term structure would allow for the construction of market-implied, forward-looking term rates via the formula $F(t, t+\delta)=\left(1 / P_{t, t+\delta}-1\right) / \delta$; an important benchmark, much coveted by market participants. The most liquid market instrument referencing RFR rates are the one-month and three-month SOFR futures contracts trading on the Chicago Mercantile Exchange. The non-linearity of the futures contract implies that futures prices do not determine the initial curve $\left(P_{0 t}\right)_{t \geq 0}$ in a unique fashion and vice versa, of course. However, model-free attempts to use futures prices to infer the SOFR-curve have been pursued by applying an approximation in Heitfield and Park (2019). The authors envisage to work on a similar procedure, but performed in an arbitrage- and approximation-free manner using the model structure presented in this paper.

A much easier and model-free approach to infer the initial curve would be possible should a liquid market for truly linear derivatives, such as swaps, arise. In this case the initial curve could be constructed using standard single-curve bootstrapping techniques.

It is unclear whether cash market participants will be satisfied with solely using a benchmark free of credit and liquidity risk premia such as SOFR. Alternative indices, such as the ICE Bank Yield Index or AMERIBOR, which include credit risk premia, might soon become increasingly relevant as an alternative to SOFR once LIBOR is discontinued. If this is what the future will bring, then the single-curve framework of this paper would need to be extended to a multi-curve setting in order to price the differences in risk premia across indices. This could be done along the lines of Crépey et al. (2016) and Macrina and Mahomed (2018), properly adjusting for the difference in model structure.

Author Contributions: A.M. and D.S. contributed equally. All authors have read and agreed to the published version of the manuscript.

Funding: This research received no external funding.

Acknowledgments: The authors are grateful to Jesper Lund Pedersen for suggestions and comments, and thank two anonymous peer-reviewers for feedback that helped improve this paper.

Conflicts of Interest: The authors declare no conflict of interest. 


\section{Appendix A. General Results}

Lemma A1. Let $U$ be a random variable and assume there exists $\delta>0$ such that $M^{U}(v):=\mathbb{E}\left[\mathrm{e}^{v U}\right]<\infty$ for all $v \in(-\delta, \delta)$. Then

$$
\mathbb{E}\left[U \mathrm{e}^{v U}\right]=\left.\frac{\partial M^{U}(v)}{\partial v}\right|_{v=v^{\prime}}, \forall v \in(-\delta, \delta) .
$$

Lemma A2. If $U>0$ is a random variable then it satisfies

$$
\mathbb{E}\left[U^{-1}\right]=\int_{0}^{\infty} \mathbb{E}\left[\mathrm{e}^{-s U}\right] \mathrm{d} s
$$

Proof. One has that, for $x>0, \int_{0}^{\infty} \mathrm{e}^{-s x} \mathrm{~d} s=1 / x$. The result is produced by applying Fubini's Theorem:

$$
\mathbb{E}\left[U^{-1}\right]=\mathbb{E}\left[\int_{0}^{\infty} \mathrm{e}^{-s U} \mathrm{~d} s\right]=\int_{0}^{\infty} \mathbb{E}\left[\mathrm{e}^{-s U}\right] \mathrm{d} s
$$

Lemma A3. Let $U>0$ and $W$ be random variables, and there exists a $\delta>0$ such that $\mathbb{E}\left[\mathrm{e}^{v_{1} U+v_{2} W}\right]<\infty$ for all $v_{1}, v_{2} \in(-\delta, \delta)$, then

$$
\mathbb{E}\left[W U^{-1}\right]=\int_{0}^{\infty} \mathbb{E}\left[W \mathrm{e}^{-s U}\right] \mathrm{d} s
$$

Proof. Observe that $\int_{0}^{\infty} \mathrm{e}^{-s x} \mathrm{~d} s=1 / x$. The result is obtained by applying Fubini's Theorem:

$$
\mathbb{E}\left[W U^{-1}\right]=\mathbb{E}\left[\int_{0}^{\infty} W \mathrm{e}^{-s U} \mathrm{~d} s\right]=\int_{0}^{\infty} \mathbb{E}\left[W \mathrm{e}^{-s U}\right] \mathrm{d} s .
$$

\section{Appendix B. Affine Processes}

Let $(\Omega, \mathcal{F}, \mathbb{Q})$ be a filtered probability space, where $\left(\mathcal{F}_{t}\right)_{0 \leq t \leq \bar{T}}$ denotes the associated filtration and $0<\bar{T}<\infty$. We set

$$
\mathcal{I}:=\left\{u \in \mathbb{R}^{d}: \sup _{t \in[0, \bar{T}]} \mathbb{E}\left[\mathrm{e}^{u \cdot X_{t}}\right]<\infty\right\}
$$

We denote by $S(\mathcal{I})$ the $\operatorname{strip} S(\mathcal{I})=\left\{z \in \mathbb{C}^{d}: \Re z \in \mathcal{I}\right\}$. We define $\mathcal{D}:=\{(t, u) \in(0, \bar{T}) \times \mathbb{C}, u \in S(\mathcal{I})\}$, and we let $\left(X_{t}\right)_{t \geq 0}$ be an affine process in the sense of definition 3.1 in Cuchiero et al. (2011), meaning that there exist functions $\phi: \mathcal{D} \rightarrow \mathbb{C}$ and $\psi: \mathcal{D} \rightarrow \mathbb{C}^{d}$ such that

$$
\mathbb{E}\left[\mathrm{e}^{u \cdot X_{t}}\right]=\exp \left(\phi_{t}(u)+\psi_{t}(u) \cdot x\right) .
$$

For all $(t, u) \in \mathcal{D}$. Since affine processes are also time-homogeneous Markov process, it follows that

$$
\mathbb{E}_{t}\left[\mathrm{e}^{u \cdot X_{T}}\right]=\exp \left(\phi_{T-t}(u)+\psi_{T-t}(u) \cdot X_{t}\right) \quad \text { for } 0 \leq t \leq T \leq \bar{T}, u \in S(\mathcal{I}) .
$$

Lemma A4. For any $v_{2} \in \operatorname{int}(\mathcal{I})$ and for $0 \leq t \leq T \leq \bar{T}$ and $\forall c_{1}, c_{2} \in \mathbb{R}$ and $\forall v_{1} \in \mathbb{R}^{d}$. It follows that

$$
\mathbb{E}_{t}\left[\left(c_{1}+v_{1} \cdot X_{T}\right) \mathrm{e}^{c_{2}+v_{2} \cdot X_{T}}\right]=\mathrm{e}^{c_{2}+\phi_{T-t}\left(v_{2}\right)+\psi_{T-t}\left(v_{2}\right) \cdot X_{t}}\left(c_{1}+v_{1} \cdot\left[\left.\frac{\partial \phi_{T-t}(u)}{\partial u}\right|_{u=v_{2}}+\left(\left.\frac{\partial \psi_{T-t}(u)}{\partial u}\right|_{u=v_{2}}\right)^{\top} X_{t}\right]\right) .
$$


Proof. Let $X_{T}^{j}$ and $u^{j}$ be an arbitrary component in $X_{T}$ and $u \in \operatorname{int}(\mathcal{I})$, respectively. Then

$$
\frac{\partial}{\partial u^{j}} \mathbb{E}_{t}\left[\mathrm{e}^{u \cdot X_{T}}\right]=\mathbb{E}_{t}\left[\frac{\partial}{\partial u^{j}} \mathrm{e}^{u \cdot X_{T}}\right]=\mathbb{E}_{t}\left[X_{T}^{j} \mathrm{e}^{u \cdot X_{T}}\right] .
$$

Differentiating within the expectation hinges, by way of the dominated convergence theorem, on the condition $\mathbb{E}_{t}\left[\left|X_{T}^{j} \mathrm{e}^{u \cdot X_{T}}\right|\right]<\infty$ which can be established by Hölder's inequality. We have

$$
\mathbb{E}_{t}\left[\left|X_{T}^{j} \mathrm{e}^{u \cdot X_{T}}\right|\right] \leq \mathbb{E}_{t}\left[\left|X_{T}^{j}\right|^{p}\right]^{1 / p} \mathbb{E}_{t}\left[\mathrm{e}^{q u \cdot X_{T}}\right]^{1 / q}, \quad p>1, \quad q=p /(p-1)
$$

Since 0 is in the interior of $\mathcal{I}$, it follows that $\mathbb{E}\left[\left|X_{T}^{j}\right|^{p}\right]<\infty$. As $p$ can be chosen arbitrarily large it follows that, since $u \in \operatorname{int}(\mathcal{I}), q$ can be made sufficiently close to 1 such that $q u \in \mathcal{I}$ and thus $\mathbb{E}_{t}\left[\mathrm{e}^{q u \cdot X_{T}}\right]<\infty$. From Inequality (A8) one then has $\mathbb{E}_{t}\left[\left|X_{T}^{j} \mathrm{e}^{u \cdot X_{T}}\right|\right]<\infty$. Applying the same arguments for all $j=1, \ldots, d$ and using Equations (A5) and (A7) we get

$$
\mathbb{E}_{t}\left[X_{T} \mathrm{e}^{v_{2} \cdot X_{T}}\right]=\left.\frac{\partial}{\partial u} \mathbb{E}_{t}\left[\mathrm{e}^{u \cdot X_{T}}\right]\right|_{u=v_{2}}=\mathrm{e}^{\phi_{T-t}\left(v_{2}\right)+\psi_{T-t}\left(v_{2}\right) \cdot X_{t}}\left(\left[\left.\frac{\partial \phi_{T-t}(u)}{\partial u}\right|_{u=v_{2}}+\left(\left.\frac{\partial \psi_{T-t}(u)}{\partial u}\right|_{u=v_{2}}\right)^{\top} X_{t}\right]\right),
$$

from which Equation (A6) follows.

Lemma A5. For all $u, v \in S(\mathcal{I})$, such that $u+\psi_{T-U}(v) \in S(\mathcal{I})$ we have for $0 \leq t \leq T \leq U$

$$
\mathbb{E}_{t}\left[\mathrm{e}^{u \cdot X_{T}+v \cdot X_{U}}\right]=\mathrm{e}^{\phi_{T-u}(v)+\phi_{T-t}\left(u+\psi_{T-U}(v)\right)+\psi_{T-t}\left(u+\psi_{T-t}(v)\right) \cdot X_{t}} .
$$

Proof. Using the tower property and Equation (A5), one has that

$$
\begin{aligned}
\mathbb{E}_{t}\left[\mathrm{e}^{u \cdot X_{T}+v \cdot X_{U}}\right] & =\mathbb{E}_{t}\left[\mathrm{e}^{u \cdot X_{T}} \mathbb{E}_{T}\left[\mathrm{e}^{u \cdot X_{U}}\right]\right] \\
& =\exp \left[\phi_{T-U}(v)+\phi_{T-t}\left(u+\psi_{T-U}(v)\right)+\psi_{T-t}\left(u+\psi_{T-t}(v)\right) \cdot X_{t}\right] .
\end{aligned}
$$

Lemma A6. Let $\left(Z_{t}\right)$ be a one dimensional square-root process, described by

$$
\mathrm{d} Z_{t}=\kappa\left(\theta-Z_{t}\right) \mathrm{d} t+\sigma \sqrt{Z_{t}} \mathrm{~d} W_{t}, \quad Z_{0}>0,
$$

where $\left(W_{t}\right)_{t \geq 0}$ is a standard Brownian motion, $\kappa, \theta$ and $\sigma$ are positive constants. The joint moment generating function is given by

$$
\mathbb{E}\left[\exp \left(u_{1} \int_{0}^{t} Z_{s} \mathrm{~d} s+u_{2} Z_{t}\right)\right]=\exp \left(A_{t}\left(u_{1}, u_{2}\right)+B_{t}\left(u_{1}, u_{2}\right) Z_{0}\right),
$$

where

$$
\begin{aligned}
& B_{t}\left(u_{1}, u_{2}\right)=\frac{u_{2}\left(\kappa+\gamma+\mathrm{e}^{\gamma t}(\gamma-k)\right)+2 u_{1}\left(\mathrm{e}^{\gamma t}-1\right)}{\left(\kappa-u_{2} \sigma^{2}\right)\left(\mathrm{e}^{\gamma t}-1\right)+\gamma\left(\mathrm{e}^{\gamma t}+1\right)} \\
& A_{t}\left(u_{1}, u_{2}\right)=\frac{2 \kappa \theta}{\sigma^{2}} \ln \left(\frac{2 \gamma(\gamma+\kappa) t / 2}{\left(\kappa-u_{2} \sigma^{2}\right)\left(\mathrm{e}^{\gamma t}-1\right)+\gamma\left(\mathrm{e}^{\gamma t}+1\right)}\right), \quad \gamma=\sqrt{\kappa^{2}-2 \sigma^{2} u_{1}},
\end{aligned}
$$

$\forall u_{1}, u_{2} \in \bar{I} \subseteq \mathcal{I}$. Here, $\mathcal{I}$ is full the strip of existence for moment generating function of the affine process $\left(\int_{0}^{t} Z_{s} d s, Z_{t}\right)_{t \geq 0}$ and $\bar{I}=\left\{\left(u_{1}, u_{2}\right) \in \mathbb{R}^{2} \mid u_{1}<\frac{k^{2}}{2 \sigma^{2}}, u_{2} \leq \frac{\kappa}{\sigma^{2}}\right\}$. 
Proof. The expressions for $A_{t}\left(u_{1}, u_{2}\right)$ and $B_{t}\left(u_{1}, u_{2}\right)$ are stated to hold for $u_{1}, u_{2} \leq 0$ in Proposition 6.3.4.1 in Jeanblanc et al. (2009). The functions $A_{t}\left(u_{1}, u_{2}\right)$ and $B_{t}\left(u_{1}, u_{2}\right)$ are verified to be analytical also for $u_{1}<k^{2} /\left(2 \sigma^{2}\right)$ and $u_{2} \leq \kappa / \sigma^{2}$. To verify that the analytical extension remains the unique moment generating function in the extended domain, Keller-Ressel and Mayerhofer (2015) show that a sufficient and necessary condition is that the functions $A_{t}\left(u_{1}, u_{2}\right)$ and $B_{t}\left(u_{1}, u_{2}\right)$ are solutions to the generalized Riccati equations associated with the affine process $\left(\int_{0}^{t} Z_{s} d s, Z_{t}\right)_{t \geq 0}$. By standard theory, see for example Duffie et al. (2000), these are given by

$$
\begin{aligned}
& d B_{t}=-\kappa B_{t}+\frac{1}{2} \sigma^{2} B_{t}^{2}+u_{1}, \quad B_{0}(0)=u_{2}, \\
& d A_{t}=\kappa \theta B_{t}, \quad A_{0}(0)=0 .
\end{aligned}
$$

Inserting the expressions $A_{t}\left(u_{1}, u_{2}\right)$ and $B_{t}\left(u_{1}, u_{2}\right)$ above and verifying that they are indeed solutions completes the proof.

\section{References}

Alfeus, Mesias, Martino Grasselli, and Erik Schlögl. 2018. A consistent stochastic model of the term structure of interest rates for multiple tenors. Journal of Economic Dynamics and Control, forthcoming.

Akahori, Jiro, Yuji Hishida, Josef Teichmann, and Takahiro Tsuchiya. 2014. A heat kernel approach to interest rate models. Japan Journal of Industrial and Applied Mathematics 31: 419-39.

Akahori, Jiro, and Andrea Macrina. 2012. Heat kernel interest rate models with time-inhomogeneous Markov processes. International Journal of Theoretical and Applied Finance 17: 1-34.

Ambrosio, Luigi, and Gianni, Dal Masio. 1990. A general chain rule for distributional derivatives. Proc. Amer. Math. Soc. 108: 691-702.

Backwell, Alex, Andrea Macrina, Erik Schlögl, and David Skovmand. 2019. Tenor-based interest rate term structures: roll-over risk perspective. Available online: https:/ / dx.doi.org/10.2139/ssrn.3399680 (accessed on 10 January 2020).

Crépey, Stéphane, Andrea Macrina, Tuyet M. Nguyen, and David Skovmand. 2016. Rational multi-curve models with counterparty-risk valuation adjustments, Quantitative Finance 16: 847-66.

Cuchiero, Christa, Damir Filipović, Eberhard Mayerhofer, and Josef Teichmann. 2011. Affine processes on positive semidefinite matrices. The Annals of Applied Probability 21: 397-463.

Döberlein, Frank, and Martin Schweizer. 2001. On savings accounts in semimartingale term structure models. Stochastic Analysis and Applications 19: 605-26.

Duffie, Darrell., Jun Pan, and Kenneth Singleton. 2000. Transform analysis and asset pricing for affine jump-diffusions. Econometrica 68: 1343-76.

Filipović, Damir, Martin Larsson, and Anders B. Trolle. 2017. Linear-rational term structure models, The Journal of Finance 72: 655-704.

Flesaker, Björn, and Lane P. Hughston. 1996. Positive interest. Risk 9: 46-9.

Gyntelberg, Jacob, and Philip. D. Wooldridge. 2008. Interbank rate fixings during the recent turmoil. In BIS Quarterly Review, March 2018. Available online: https:/ /www.bis.org/publ/qtrpdf/r_qt0803g.pdf (accessed on 7 January 2020).

Henrard, Marc P. 2019. LIBOR fallback and quantitative finance. Risks 7: 88.

Heitfield, Erik, and Yang-Ho Park. 2019. Inferring term rates from SOFR futures prices. Finance and Economics Discussion Series 2019-014. Washington: Board of Governors of the Federal Reserve System. Available online: https:/ / doi.org/10.17016/FEDS.2019.014 (accessed on 15 January 2020).

Hunt, Philip, and Joanne Kennedy. 2004. Financial Derivatives in Theory and Practice. Hoboken: Wiley.

ISDA. 2019. Summary of Responses to the ISDA Supplemental Consultation on Spread and Term Adjustments. Available online: https://www.isda.org/a/0LPTE/2019.09.18-Anonymized-ISDASupplemental-Consultation-Report.pdf (accessed on 11 January 2020).

Jeanblanc, Monique, Marc Yor, and Marc Chesney. 2009. Mathematical Methods for Financial Markets. Berlin: Springer Science \& Business Media. 
Keller-Ressel, Martin, 2008. Affine processes: theory and applications in finance. Ph.D. thesis, TU Vienna, Vienna, Austria.

Keller-Ressel, Martin, and Eberhard Mayerhofer. 2015 . Exponential moments of affine processes. The Annals of Applied Probability 25: 714-52.

Lyashenko, Andrei, and Fabio Mercurio. 2019. Looking forward to backward-looking rates: A modeling framework for term rates replacing LIBOR. Available online: https://dx.doi.org/10.2139/ssrn.3330240 (accessed on 19 December 2019).

Macrina, Andrea. 2014. Heat kernel models for asset pricing. International Journal of Theoretical and Applied Finance 17: 1-34.

Macrina, Andrea, and Obeid Mahomed. 2018. Consistent valuation across curves using pricing kernels. Risk 6: 1-32.

Mercurio, Fabio. 2018. A simple multi-curve model for pricing SOFR futures and other derivatives. Available online: http:/ / dx.doi.org/10.2139/ssrn.3225872 (accessed on 15 January 2020).

Miltersen, Kristian R., Klaus Sandmann, and Dieter Sondermann. 1997. Closed form solutions for term structure derivatives with log-normal interest rates. The Journal of Finance 52: 409-30.

Nguyen, The, and Frank T. Seifried. 2015. The affine rational potential model. Available online: http://ssrn.com/ abstract $=\mathrm{t}=2707081$ (accessed on 17 January 2020).

Rutkowski, M. 1997. A note on the Flesaker-Hughston model of the term structure of interest rates. Applied Mathematical Finance 4: 151-63.

Singleton, K. J., and L. Umantsev. 2002. Pricing coupon-bond options and swaptions in affine term structure models. Mathematical Finance 12: 427-46.

(C) 2020 by the authors. Licensee MDPI, Basel, Switzerland. This article is an open access article distributed under the terms and conditions of the Creative Commons Attribution (CC BY) license (http:/ / creativecommons.org/licenses/by/4.0/). 